TRANSACTIONS OF THE

AMERICAN MATHEMATICAL SOCIETY

Volume 363, Number 8, August 2011, Pages 4285-4307

S 0002-9947(2011)05356-0

Article electronically published on March 22, 2011

\title{
A UNIFORM ESTIMATE FOR POSITIVE SOLUTIONS OF SEMILINEAR ELLIPTIC EQUATIONS
}

\author{
G. FUSCO, F. LEONETTI, AND C. PIGNOTTI
}

\begin{abstract}
We consider the semilinear elliptic equation $\Delta u=W^{\prime}(u)$ with Dirichlet boundary condition in a Lipschitz, possibly unbounded, domain $\Omega \subset$ $\mathbb{R}^{n}$. Under suitable assumptions on the potential $W$, we deduce a condition on the size of the domain that implies the existence of a positive solution satisfying a uniform pointwise estimate. Here uniform means that the estimate is independent of $\Omega$.

Under some geometric restrictions on the domain, we extend the analysis to the case of mixed Dirichlet-Neumann boundary conditions.

As an application of our estimate we give a proof of the existence of potentials such that, independent of the choice of $\Omega$ and of the value of $\lambda>0$, the equation $\Delta u=\lambda W^{\prime}(u)$ has infinitely many positive solutions.
\end{abstract}

\section{INTRODUCTION}

We consider the semilinear elliptic problem

$$
\begin{cases}\Delta u=W^{\prime}(u), & x \in \Omega, \\ u=0, & x \in \partial \Omega, \\ u>0, & x \in \Omega,\end{cases}
$$

where $\Omega \subset \mathbb{R}^{n}, n \geq 1$, is a domain with nonempty Lipschitz boundary. We assume that $W: \mathbb{R} \rightarrow \mathbb{R}$ is a $C^{2}$ function satisfying

(a) There exists a constant $\mu>0$ such that

$$
\begin{gathered}
0=W(\mu)<W(s), s \in[0,+\infty), s \neq \mu, \\
W(-s) \geq W(s), s \in[0,+\infty) ;
\end{gathered}
$$

(b) $W^{\prime}(s) \leq 0, \quad s \in(0, \mu)$;

(c) $W^{\prime \prime}(\mu)>0$.

Under these conditions on $W$, if $\Omega$ is bounded, existence of a solution for problem (1.1) can be proved by the method of sub and sup solutions or by minimization of the energy functional associated to (1.1). We refer for instance to [10, 17, [15. We derive a condition on the size of $\Omega$ that, independent of $\Omega$ being bounded or unbounded, ensures existence of a solution of problem (1.1) which satisfies a pointwise estimate which is uniform in the sense that is valid for all domains.

For $x \in \mathbb{R}^{n}, \rho>0$, we let

$$
B_{\rho}(x)=\left\{y \in \mathbb{R}^{n}:|y-x|<\rho\right\}, \quad B_{\rho}=B_{\rho}(0) .
$$

Received by the editors November 2, 2009.

2010 Mathematics Subject Classification. Primary 35J61, 35B09.

Key words and phrases. Semilinear elliptic equations, positive solutions.

(C)2011 American Mathematical Society 
Moreover, we set

$$
\begin{gathered}
\lambda A=\{\lambda x: x \in A\}, \quad A \subset \mathbb{R}^{n}, \lambda>0, \\
A+B=\{x+y: x \in A, y \in B\}, \quad A, B \subset \mathbb{R}^{n}
\end{gathered}
$$

and denote $d(x, E)$ as the Euclidean distance of the point $x \in \mathbb{R}^{n}$ from the set $E \subset \mathbb{R}^{n}$. Our main result is the following:

Theorem 1.1. Assume $\Omega$ and $W$ as above. Then there are positive constants $R^{*}, k, K$, depending only on $W$ and $n$, such that if $\Omega$ contains a closed ball of radius $R^{*}$, then problem (1.1) has a positive solution $u \in C^{2}(\Omega) \cap C(\bar{\Omega})$ verifying

$$
\begin{gathered}
0<u(x)<\mu, \quad x \in \Omega, \\
\mu-u(x) \leq K e^{-k d(x, \partial \Omega)}, \quad x \in \Omega .
\end{gathered}
$$

In addition, there are $r^{*} \in\left(0, R^{*}\right)$ and $a \in(0, \mu)$ depending only on $W$ and $n$, such that

$$
\mu-a<u(x), \quad x \in \Omega_{R^{*}}+B_{r^{*}},
$$

where

$$
\Omega_{R^{*}}=\left\{x \in \Omega: d(x, \partial \Omega)>R^{*}\right\} .
$$

Remark 1.2. Without straightening the assumptions in Theorem 1.1, uniqueness of positive solutions cannot be expected. A sufficient condition for uniqueness is $W^{\prime}(s) / s$ being nondecreasing in $(0, \mu)$ (see [7]).

Remark 1.3. If $\Omega$ is invariant under a group $G$ of orthogonal transformations, then, assuming uniqueness, we can also assume that the solution $u$ given by Theorem 1.1 is invariant under $G$; that is, $u(g x)=u(x), \forall g \in G$.

Remark 1.4. If $W^{\prime}(0)=0$, equation (1.1) has the trivial solution $u \equiv 0$. If problem (1.1) is considered in $\Omega=\lambda \tilde{\Omega}, \lambda>0$, where $\tilde{\Omega}$ is a fixed domain, then there is a bifurcation value $\lambda_{c}$ of $\lambda$ such that, for $\lambda \leq \lambda_{c}, u \equiv 0$ is the only solution, while for $\lambda>\lambda_{c}$ there also exists a positive solution. The condition that $\Omega$ contains a closed ball of radius $R^{*}$ is a condition on the size of $\Omega$ ensuring that the regime after bifurcation prevails. The value $\lambda_{R^{*}}$ of $\lambda$, such that, for $\lambda>\lambda_{R^{*}}, \lambda \tilde{\Omega}$ contains a ball of radius $R^{*}$, yields an upper bound for the critical value $\lambda_{c}$.

Remark 1.5. The monotonicity assumption (b) can be relaxed. The assumption $W^{\prime \prime}(\mu)>0$ implies the existence of numbers $a, c>0$ such that

$$
W^{\prime}(\mu-s) \leq-c^{2} s, \quad s \in[0, a] .
$$

Then, as we will show at the end of section 2, we can replace (b) by the weaker condition

$$
W^{\prime}(s)<\frac{c^{2} a^{2}}{2(\mu-a)},
$$

without affecting the conclusions of Theorem 1.1. We note that (1.7) is compatible with potentials that have various maxima and minima in the interval $[0, \mu)$; in particular with potentials having a strict local minimum at 0 . For instance for the potential

$$
W(s)=\left(1-s^{2}\right)^{2}+\eta q \tanh ^{2} \frac{s}{q}, \quad \eta, q>0
$$


we have $W^{\prime}(s)<\eta$, and, if $\eta$ and $q$ are sufficiently small, $W$ satisfies condition (1.7) and has a strict local minimum at 0 . If $W$ has $N>1$ local minima $W\left(\mu_{1}\right)>\cdots>$ $W\left(\mu_{N}\right)$ at $0<\mu_{1}<\cdots<\mu_{N}$ and (1.6), (1.7) hold for some $c_{j}, a_{j}>0, j=1, \ldots, N$, then by applying Theorem 1.1 in each interval $\left[0, \mu_{j}\right], j=1, \ldots, N$, we get the existence of $N$ distinct positive solutions with the corresponding estimates (1.2), (1.3) in any domain $\Omega$ that contains a closed ball of radius $\tilde{R}^{*}=\max _{j=1, \ldots, N} R_{j}^{*}$, where $R_{j}^{*}$ is the value of $R^{*}$ corresponding to $\left.W\right|_{\left[0, \mu_{j}\right]}$. An example of $W$ that satisfies these conditions is

$$
W(s)=\sum_{j=1}^{N} C_{j} \chi_{[2(j-1), 2 j]} g(s+1-2 j),
$$

where $\chi_{E}$ denotes the characteristic function of a set $E, g:[-1,1] \rightarrow \mathbb{R}$ is a function of the form $g(s)=-\left(1-s^{2}\right)^{4}$ and the constants $C_{j}>0$ are chosen to have the ratio $C_{j+1} / C_{j}, j=1, \ldots, N-1$, sufficiently large.

The arguments in the proof of Theorem 1.1 can be used for giving a simple proof of the existence of a potential function such that the equation

$$
\left\{\begin{array}{l}
\Delta u=\lambda W^{\prime}(u), \quad x \in \Omega, \\
u=0, \quad x \in \partial \Omega
\end{array}\right.
$$

has infinitely many solutions for any $\lambda>0$ and for any domain. This was proved in [11] for a general class of nonlinear elliptic equations including (1.9) and for bounded domains. See also [9, 17, 14] for related results. We prove the following

Theorem 1.6. Assume $\Omega$ as in Theorem 1.1 and let $W:[0,+\infty) \rightarrow \mathbb{R}$ be a nonincreasing $C^{1,1}$ function such that there exist sequences $a_{j}, c_{j}, \mu_{j}, j \in \mathbb{N}$, with

$$
\begin{gathered}
\lim _{j \rightarrow \infty} a_{j}=\lim _{j \rightarrow \infty} c_{j}=\lim _{j \rightarrow \infty} \mu_{j}=+\infty, \\
W^{\prime}\left(\mu_{j}\right)=0, \quad \lim _{s \rightarrow 0^{+}} W^{\prime \prime}\left(\mu_{j}-s\right)=c_{j}^{2}, \\
\limsup _{j \rightarrow+\infty} \frac{\mu_{j}-a_{j}}{a_{j}}<+\infty .
\end{gathered}
$$

Then, the semilinear elliptic problem (1.9) admits infinitely many distinct positive solutions independent of the value of $\lambda>0$ and independent of the choice of the domain $\Omega$.

Remark 1.7. The assumptions in the above theorem are incompatible with $W$ being $C^{2}$. Nevertheless the conclusions of Theorem 1.1 remain valid when $W \mid\left[0, \mu_{j}\right], j=$ $1,2, \ldots$, satisfies the slightly weaker smoothness requirement in Theorem 1.6.

We also consider the mixed Dirichlet-Neumann problem

$$
\begin{cases}\Delta u=W^{\prime}(u), & x \in \Omega, \\ u=0, & x \in \Gamma_{D}, \\ \frac{\partial u}{\partial \nu}=0, & x \in \Gamma_{N}, \\ u>0, & x \in \Omega,\end{cases}
$$

where $\Omega \subset \mathbb{R}^{n}$ is a $C^{2}$ open connected set, $\Gamma_{D} \subset \partial \Omega$ and $\Gamma_{N}=\partial \Omega \backslash \bar{\Gamma}_{D}$ are nonempty relatively open $(n-1)$-dimensional smooth submanifolds of $\partial \Omega$.

Given $\rho>0$ and $x \in \bar{\Omega}$, let $\mathcal{B}_{\rho}(x)$ be the connected component of $B_{\rho}(x) \cap \Omega$ such that $x \in \overline{\mathcal{B}}_{\rho}(x)$. We also set $\partial_{\Omega} \mathcal{B}_{\rho}(x)=\partial \mathcal{B}_{\rho}(x) \cap \partial \Omega$. 


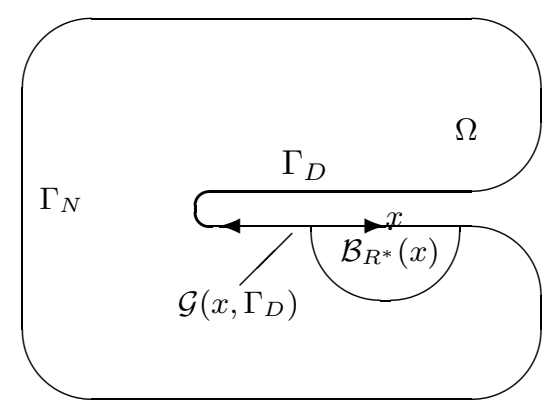

Figure $1 . \Omega, \Gamma_{D}$ and $\Gamma_{N}$ with $\Gamma_{N}$ convex

Definition 1.8. $\Gamma_{N}$ is said to be convex if for each $x \in \Gamma_{N}$ there is $\rho>0$ such that $\mathcal{B}_{\rho}(x)$ is convex.

For $x \in \bar{\Omega}$ we define the geodesic distance of $x$ from $\Gamma_{D}, \mathcal{G}\left(x, \Gamma_{D}\right)$ by setting

$$
\mathcal{G}\left(x, \Gamma_{D}\right)=\inf _{\gamma\left(x, \Gamma_{D}\right)} \mathcal{H}^{1}\left(\gamma\left(x, \Gamma_{D}\right)\right),
$$

where $\mathcal{H}^{1}$ is the one-dimensional Hausdorff measure and the infimum is taken on the set of the absolutely continuous paths $\gamma\left(x, \Gamma_{D}\right) \subset \bar{\Omega}$ joining $x$ to $\Gamma_{D}$.

We have the following theorem.

Theorem 1.9. Assume $W$ satisfies (a), (b), (c) and $\Omega \subset \mathbb{R}^{n}$ is a $C^{2}$ open connected set with $\Gamma_{D} \subset \partial \Omega, \Gamma_{N}=\partial \Omega \backslash \bar{\Gamma}_{D}$ as above. Moreover, assume that $\Gamma_{N}$ is convex in the sense of Definition 1.8 and let $R^{*}, k, K$ as in Theorem 1.1. Then the existence of $x \in \bar{\Omega}$ such that $\overline{\mathcal{B}_{R^{*}}(x)} \cap \bar{\Gamma}_{D}=\emptyset$ implies that problem (1.13) has a positive solution $u \in C^{2}(\Omega) \cap C(\bar{\Omega})$. Moreover,

$$
\begin{gathered}
0<u(x)<\mu, \quad \forall x \in \Omega, \\
\mu-u(x) \leq K e^{-k \mathcal{G}\left(x, \Gamma_{D}\right)}, \quad \forall x \in \Omega .
\end{gathered}
$$

In addition, it results in

$$
\mu-a<u(x), \quad x \in \bigcup_{y \in \bar{\Omega}: \partial_{\Omega} \mathcal{B}_{R^{*}}(y) \subset \Gamma_{N}} \mathcal{B}_{r^{*}}(y),
$$

with $r^{*}$ and $a$ as in Theorem 1.1 .

Figure 1 shows an example where $\Gamma_{N}$ is convex in the sense of Definition 1.8 , Notice the difference between $d\left(x, \Gamma_{D}\right)$ and $\mathcal{G}\left(x, \Gamma_{D}\right)$. If $\Gamma_{N}$ is not convex, the shape of $\Gamma_{N}$ can be quite involved and the imposing Neumann boundary condition may limit the decay rate of the solution. In section 4 we derive an exponential estimate which takes into account the geometry of $\Gamma_{N}$ (cf. Theorem 5.2).

Our approach to the proof of Theorems 1.1 and 1.9 is variational and uses ideas developed in [2] and [3] (see also [4]) in the context of semilinear elliptic systems. We first consider the case of a bounded domain and find $u$ as a minimizer of the action functional

$$
\mathcal{E}(u, \Omega)=\int_{\Omega}\left\{\frac{1}{2}|\nabla u|^{2}+W(u)\right\} d x, \quad u \in W_{0}^{1,2}(\Omega),
$$


on a suitable set $\mathcal{A} \subset W_{0}^{1,2}(\Omega)$ of admissible functions. Here, as usual, $W_{0}^{1,2}(\Omega)$ denotes the set of the functions of $W^{1,2}(\Omega)$ vanishing on $\partial \Omega$.

By constructing judicious comparison functions we also establish the estimates in (1.3) and (1.16). Once the exponential estimate is available, a standard limit procedure based on elliptic regularity allows us to extend the existence result and the estimates to the case of unbounded domains.

We conclude this introduction by observing that, under the additional assumption that $W$ is even,

$$
W(-s)=W(s), \quad s \in \mathbb{R},
$$

by means of Theorem 1.1 we can derive the existence of various entire solutions for the problem

$$
\Delta u=W^{\prime}(u), \quad x \in \mathbb{R}^{n} .
$$

1. Saddle solutions. Assume $W(s)=\left(1-s^{2}\right)^{2}, n=2 m, m \in \mathbb{N}$. Following [6] we say that a bounded solution $u: \mathbb{R}^{n} \rightarrow \mathbb{R}$ is a saddle solution if

(h1) $u\left(g_{1} x, g_{2} y\right)=u(x, y), \quad x, y \in \mathbb{R}^{m}, \quad g_{1}, g_{2} \in O(m)$;

(h2) $u(y, x)=-u(x, y), \quad x, y \in \mathbb{R}^{m}$;

(h3) $u(x, y)>0$, on $\Omega=\{(x, y):|x|>|y|\}$.

Note that (h1), (h2) and (h3) imply that a saddle solution vanishes on the Simon cone $C=\{(x, y):|x|=|y|\}$. Saddle solutions have been studied in detail in [7] for $m=1$, see also [16], and for general $m$ in [6]. To prove existence (for $m>1$ ) we use Theorem 1.1 with $\Omega=\{|x|>|y|\} 1$ and restrict to the weakly closed subspace $\hat{W}_{0}^{1,2}(\Omega)=\left\{u \in W_{0}^{1,2}(\Omega): u\left(g_{1} x, g_{2} y\right)=u(x, y), \quad x, y \in \Omega, \quad g_{1}, g_{2} \in O(m)\right\}$. This yields a solution $u: \Omega \rightarrow \mathbb{R}$ that satisfies (h1) and (h3) and the exponential estimate (1.3). Then, the solution is extended to the whole of $\mathbb{R}^{n}$ by odd reflection across the Simon cone. If $m=1$ we take $\Omega=\{x>|y|\}$, and we get a saddle solution by odd reflection first with respect to $y=x$ and then with respect to $y=-x$.

The paper [1] studies saddle type solutions $u_{n}: \mathbb{R}^{2} \rightarrow \mathbb{R}$ that vanish on the lines $l_{k}=\left\{(x, y) \in \mathbb{R}^{2}: y=x \tan (k \pi / n)\right\}, k=0,1, \ldots, 2 n-1$. Under the assumption (1.19) the existence of a solution $u_{n}$ with nodal lines $l_{k}, k=0,1, \ldots, 2 n-1$, can be derived by Theorem 1.1 with $\Omega$ the sector $\left\{(x, y) \in \mathbb{R}^{2}: 0<y<x \tan (\pi / n), x>\right.$ $0\}$. The solution given by Theorem 1.1 is then extended to the whole $\mathbb{R}^{2}$ by odd reflection across the lines $l_{k}$.

We can also consider "tick" saddle solutions. Apply Theorem 1.1, with $\Omega \subset$ $\mathbb{R}^{2 m+1}$ defined by

$$
\Omega=\left\{(x, y, z) \in \mathbb{R}^{m} \times \mathbb{R}^{m} \times \mathbb{R}:|x|>|y|,-D<z<D\right\} .
$$

Under the assumption $D>R^{*}$ Theorem 1.1 yields a positive solution $u: \Omega \rightarrow \mathbb{R}$ to problem (1.1) that vanishes on $\partial \Omega=\{|x|=|y|, z \in[-D, D]\} \cup(\{|x|>|y|\} \times$ $\{-D, D\})$. By odd reflection with respect to $\{|x|=|y|, z \in(-D, D)\}$ and then with respect to the planes $z=(2 k+1) D, k \in \mathbb{Z}$, the solution $u: \Omega \rightarrow \mathbb{R}$ given by Theorem 1.1 can be extended to the whole of $\mathbb{R}^{2 m+1}$, yielding an entire solution of (1.20) which has a saddle structure on each plane $z=$ const and is periodic of period $4 D$ in the $z$ variable.

\footnotetext{
${ }^{1}$ The set $\Omega=\{|x|>|y|\}$ is not Lipschitz at the origin. This does not limit the validity of Theorem 1.1
} 
2. $n$-dimensional lattice. Take $\Omega \subset \mathbb{R}^{n}$ as the rectangle $\Omega=\prod_{h=1}^{n}\left(-D_{h}, D_{h}\right)$, $D_{h}>0$. Then, assuming $\min _{h} D_{h}>R^{*}$, Theorem 1.1 yields a positive solution $u: \Omega \rightarrow \mathbb{R}$. By odd reflection with respect to the planes $x_{h}=(2 k+1) D_{h}, k \in \mathbb{Z}$, $h=1, \ldots, n$, we obtain an entire solution to (1.20) which is $4 D_{h}$ periodic in the variable $x_{h}$ and is positive or negative in the rectangle

$$
\Pi_{h=1}^{n}\left(\left(2 k_{h}-1\right) D_{h},\left(2 k_{h}+1\right) D_{h}\right),
$$

depending on whether $\sum_{h=1}^{n} k_{h}$ is even or odd.

3. Theorem 1.1 can be applied to get existence and estimates to various other situations. For instance, in the case considered in [5] of an unbounded domain $\Omega$ defined by

$$
\Omega=\left\{x \in \mathbb{R}^{n}: x_{n}>\phi\left(x_{1}, \ldots, x_{n-1}\right)\right\},
$$

where $\phi: \mathbb{R}^{n-1} \rightarrow \mathbb{R}$ is a Lipschitz continuous function, Theorem 1.1 yields a positive solution.

The paper is organized as follows. In section 2 we present the comparison lemmas that we use for deriving the exponential estimate (1.3). In section 3 we prove Theorem 1.1 for the case of a bounded domain and then extend the result to unbounded domains. In section 4 we discuss the case of Dirichlet-Neumann boundary conditions under a convexity assumption on $\Gamma_{N}$. Finally, in section [5 we consider the mixed problem in more general domains.

\section{BASIC LEMMAS}

In this section we give some lemmas that we will use to prove Theorem 1.1

We set

$$
u=\mu-v \quad \text { and } \quad V(v)=W(\mu-v) .
$$

Then, we can rewrite for $V$ properties $(\mathbf{a}),(\mathbf{b}),(\mathbf{c})$ of $W$ as follows:

(a1) $0=V(0)<V(s), s \in(-\infty, \mu], s \neq 0$ $V(\mu+s) \geq V(\mu-s), \quad s \in[0,+\infty)$

(b1) $V^{\prime}(s) \geq 0, \quad s \in(0, \mu)$;

(c1) $V^{\prime \prime}(0)>0$.

Moreover, equation (1.1) is equivalent to

$$
\begin{cases}\Delta v=V^{\prime}(v), & x \in \Omega \\ v=\mu, & x \in \partial \Omega, \\ v<\mu, & x \in \Omega .\end{cases}
$$

Let $O \subset \mathbb{R}^{n}$ be an open set. The action functional takes the form

$$
\mathcal{E}(v, O):=\int_{O}\left\{\frac{1}{2}|\nabla v|^{2}+V(v)\right\} d x, \quad v \in W^{1,2}(O) .
$$

Lemma 2.1. Let $O \subset \mathbb{R}^{n}$ be an open set and let $v \in W^{1,2}(O)$. Define $\tilde{v}: O \rightarrow \mathbb{R}$ as

$$
\tilde{v}(x)= \begin{cases}v(x) & \text { if } v(x) \in[0, \mu], \\ 0 & \text { if } v(x) \in(-\infty, 0) \cup(2 \mu,+\infty), \\ 2 \mu-v(x) & \text { if } v(x) \in(\mu, 2 \mu] .\end{cases}
$$

Then, $\tilde{v} \in W^{1,2}(O), 0 \leq \tilde{v} \leq \mu$ and $\mathcal{E}(\tilde{v}, O) \leq \mathcal{E}(v, O)$. 
Proof. From the definition of (2.4) it immediately follows that $\tilde{v}(x) \in[0, \mu]$, for any $x \in O$. Moreover,

$$
|\nabla \tilde{v}(x)| \leq|\nabla v(x)| \text { a.e. } x \in O,
$$

and properties (a1), (b1) of $V$ give

$$
V(\tilde{v}(x)) \leq V(v(x)) \text { a.e. } x \in O .
$$

Then, from (2.5) and (2.6) we have $\mathcal{E}(\tilde{v}, O) \leq \mathcal{E}(v, O)$.

We remark that from Lemma 2.1 it follows that it suffices to specify $W$ only in the interval $[0, \mu]$.

Let $c, a$ be as in (1.6) and let $r<R$ be positive constants.

Denote by $\varphi^{r}$ and $\psi^{r, R}$ (for short $\varphi$ and $\psi$ ) the solutions of the problems

$$
\begin{cases}\Delta \varphi=c^{2} \varphi, & x \in B_{r}, \\ \varphi=a, & x \in \partial B_{r}\end{cases}
$$

and

$$
\left\{\begin{array}{l}
\Delta \psi=0, \quad x \in B_{R} \backslash \bar{B}_{r}, \\
\psi=a, \quad x \in \partial B_{r}, \\
\psi=\mu, \quad x \in \partial B_{R} .
\end{array}\right.
$$

It is well known that the functions $\varphi^{r}$ and $\psi^{r, R}$ are radial: $\varphi^{r}(x)=\Phi^{r}(|x|)$ and $\psi^{r, R}(x)=\Psi^{r, R}(|x|)$ for some functions $\Phi^{r}:[0, r] \rightarrow \mathbb{R}$ and $\Psi^{r, R}:[r, R] \rightarrow \mathbb{R}$. Moreover, $\Phi^{r, R}$ and $\Psi^{r, R}$ are increasing; $\Phi^{r, R}$ is convex and $\Psi^{r, R}$ is concave. In the following we will often drop the superscripts $r$ and $r, R$.

Lemma 2.2. Assume $a, c>0$ as in (1.6) and let $v \in W^{1,2}\left(B_{r}\right)$ satisfy

$$
0 \leq v \leq a, x \in \bar{B}_{r}
$$

$$
|E|>0, \quad \text { where } E=\left\{x \in B_{r}: v(x)>\varphi(x)\right\} .
$$

Then there is a function $\tilde{v} \in W^{1,2}\left(B_{r}\right)$ verifying

$$
\left\{\begin{array}{l}
\tilde{v}=v, \quad x \in B_{r} \backslash E \\
\tilde{v} \leq \varphi, \quad x \in E
\end{array}\right.
$$

and

$$
\mathcal{E}\left(\tilde{v}, B_{r}\right)<\mathcal{E}\left(v, B_{r}\right) .
$$

Proof. From (2.7), integrating by parts, we have

$$
\int_{B_{r}}\left(\nabla \varphi \nabla \eta+c^{2} \varphi \eta\right) d x=0, \quad \forall \eta \in W_{0}^{1,2}\left(B_{r}\right) .
$$

In particular, if we take

$$
\eta(x)= \begin{cases}0 & \text { for } x \in B_{r} \backslash E \\ v(x)-\varphi(x) & \text { for } x \in E\end{cases}
$$

from (2.13) it follows that

$$
\int_{E}\left\{\nabla \varphi(\nabla v-\nabla \varphi)+c^{2} \varphi(v-\varphi)\right\} d x=0 .
$$


Given $s_{0} \in[0, a)$ set

$$
U_{s_{0}}(s):=V\left(s_{0}\right)+c^{2} s_{0}\left(s-s_{0}\right), \quad s \in\left(s_{0}, a\right],
$$

and observe that (1.6) implies

$$
V(s)>U_{s_{0}}(s), \quad \forall s \in\left(s_{0}, a\right], \forall s_{0} \in[0, a) .
$$

Let $\tilde{v}$ be defined by (2.11) with the equality sign in the second line of (2.11). Then we have

$$
\begin{aligned}
\mathcal{E}\left(v, B_{r}\right)- & \mathcal{E}\left(\tilde{v}, B_{r}\right)=\frac{1}{2} \int_{E}\left(|\nabla v|^{2}-|\nabla \varphi|^{2}\right) d x+\int_{E}[V(v)-V(\varphi)] d x \\
& =\frac{1}{2} \int_{E}|\nabla v-\nabla \varphi|^{2} d x+\int_{E} \nabla \varphi(\nabla v-\nabla \varphi) d x+\int_{E}[V(v)-V(\varphi)] d x \\
& =\frac{1}{2} \int_{E}|\nabla v-\nabla \varphi|^{2} d x-\int_{E} c^{2} \varphi(v-\varphi) d x+\int_{E}[V(v)-V(\varphi)] d x \\
& =\frac{1}{2} \int_{E}|\nabla v-\nabla \varphi|^{2} d x+\int_{E}\left[V(v)-U_{\varphi}(v)\right] d x,
\end{aligned}
$$

where we have used (2.15) and (2.16). Since we have $\varphi<v \leq a$ a.e. in $E$, from (2.17) we deduce

$$
V(v)-U_{\varphi}(v)>0 \quad \text { on } E .
$$

Therefore, (2.12) follows from (2.18).

Lemma 2.3. Let $v \in W^{1,2}\left(B_{R} \backslash \overline{B_{r}}\right)$ satisfy

$$
\begin{gathered}
0 \leq v \leq \mu, x \in B_{R} \backslash \bar{B}_{r}, \\
v \leq \psi, x \in \partial\left(B_{R} \backslash \bar{B}_{r}\right), \\
|E|>0, \quad \text { where } E=\left\{x \in B_{R} \backslash \bar{B}_{r}: v(x)>\psi(x)\right\} .
\end{gathered}
$$

Then there is a function $\tilde{v} \in W^{1,2}\left(B_{R} \backslash \bar{B}_{r}\right)$ such that

$$
\left\{\begin{array}{l}
\tilde{v}=v, \quad x \in\left(B_{R} \backslash \bar{B}_{r}\right) \backslash E, \\
\tilde{v} \leq \psi, \quad x \in E
\end{array}\right.
$$

and

$$
\mathcal{E}\left(\tilde{v}, B_{R} \backslash \bar{B}_{r}\right) \leq \mathcal{E}\left(v, B_{R} \backslash \bar{B}_{r}\right) .
$$

Proof. From (2.8), integrating by parts, we have

$$
\int_{B_{R} \backslash \bar{B}_{r}} \nabla \psi \nabla \eta d x=0, \quad \forall \eta \in W_{0}^{1,2}\left(B_{R} \backslash \bar{B}_{r}\right) .
$$

In particular, if we take

$$
\eta(x)= \begin{cases}0 & \text { for } x \in\left(B_{R} \backslash \bar{B}_{r}\right) \backslash E, \\ v(x)-\psi(x) & \text { for } x \in E,\end{cases}
$$

from (2.24) it follows that

$$
\int_{E} \nabla \psi(\nabla v-\nabla \psi) d x=0 .
$$


Let $\tilde{v}$ be defined by (2.22) with the equality sign in the second line of (2.22). Then we have

$$
\begin{aligned}
\mathcal{E}\left(v, B_{R} \backslash\right. & \left.\bar{B}_{r}\right)-\mathcal{E}\left(\tilde{v}, B_{R} \backslash \bar{B}_{r}\right)=\frac{1}{2} \int_{E}\left(|\nabla v|^{2}-|\nabla \psi|^{2}\right) d x+\int_{E}[V(v)-V(\psi)] d x \\
& =\frac{1}{2} \int_{E}|\nabla v-\nabla \psi|^{2} d x+\int_{E} \nabla \psi(\nabla v-\nabla \psi) d x+\int_{E}[V(v)-V(\psi)] d x \\
& =\frac{1}{2} \int_{E}|\nabla v-\nabla \psi|^{2} d x+\int_{E}[V(v)-V(\psi)] d x \geq 0
\end{aligned}
$$

where we have used (2.26), (2.19) and assumption (b1) on $V$.

Lemma 2.4. Let $\Phi=\Phi^{r}$ as before. Then, $\Phi^{\prime}(r)=\left.\Phi^{\prime}(s)\right|_{s=r}$ is strictly increasing in $(0,+\infty)$ and

$$
\lim _{r \rightarrow+\infty} \Phi^{\prime}(r)=c a .
$$

Moreover, there exists a strictly increasing function $h:(0,+\infty) \rightarrow(0,+\infty)$ such that

$$
\Phi(s) \leq e^{h(r)(s-r)} \Phi(r), \quad s \in[0, r],
$$

and

$$
\lim _{r \rightarrow \infty} h(r)=c .
$$

Proof. Set $w(s)=\Phi^{\prime}(s) / \Phi(s)$. Then, (2.7) and $\Phi^{\prime}(0)=0$ imply that $w$ solves

$$
\left\{\begin{array}{l}
w^{\prime}=c^{2}-\frac{n-1}{s} w-w^{2} \\
w(0)=0
\end{array}\right.
$$

The right hand side $g(s, w)$ of (2.31) has a positive root

$$
z(s)=\frac{1-n}{2 s}+\sqrt{\left(\frac{n-1}{2 s}\right)^{2}+c^{2}}
$$

and

$$
\operatorname{sgn} g(s, w)=\operatorname{sgn}(z(s)-w) .
$$

It results in

while (2.7) implies

$$
\lim _{s \rightarrow 0^{+}} z(s)=0 \quad \text { and } \lim _{s \rightarrow 0^{+}} z^{\prime}(s)=\frac{c^{2}}{n-1},
$$

and therefore

$$
\Delta \varphi(0)=n \Phi^{\prime \prime}(0)=c^{2} \Phi(0)
$$

follows that

$$
w^{\prime}(0)=\frac{c^{2}}{n} \text {. }
$$

$$
\left\{\begin{array}{l}
w(0)=z(0)=0 \\
w^{\prime}(0)<z^{\prime}(0)
\end{array}\right.
$$

which shows that the curve $s \rightarrow w(s)$ starts below the curve $s \rightarrow z(s)$, and by consequence we conclude that

$$
w(s)<z(s), \quad s \in(0,+\infty) .
$$


Indeed, the curves $s \rightarrow w(s)$ and $s \rightarrow z(s)$ cannot cross since $z(s)$ is strictly increasing in $(0,+\infty)$ and $g(s, z(s))=0$ from (2.32). The inequalites (2.35) and (2.33) yield

$$
\left\{\begin{array}{l}
w^{\prime}(s)>0, \quad s \in(0,+\infty) \\
\lim _{s \rightarrow+\infty} w(s)=\lim _{s \rightarrow+\infty} z(s)=c .
\end{array}\right.
$$

From (2.36) we deduce that $\Phi^{\prime}(r)=w(r) a$ is strictly increasing in $(0,+\infty)$ and that (2.28) holds.

To prove (2.29) we set

$$
h(r)=\frac{1}{r} \int_{0}^{r} w(s) d s .
$$

Then, by (2.36), $h$ is increasing and satisfies (2.30). Let $\tilde{\Phi}(s)$ be the right hand side of (2.29). Then,

$$
\frac{\Phi(s)}{\tilde{\Phi}(s)}=e^{\int_{r}^{s}[w(\tau)-h(r)] d \tau} \leq 1, \quad s \in[0, r] .
$$

Indeed, (2.37) and $h^{\prime}(r)>0$ imply that

$$
\begin{aligned}
\int_{r}^{s}[w(\tau) & -h(r)] d \tau=\int_{r}^{s} w(\tau) d \tau-\frac{s-r}{r} \int_{0}^{r} w(\tau) d \tau \\
& =\int_{0}^{s} w(\tau) d \tau-\frac{s}{r} \int_{0}^{r} w(\tau) d \tau=s[h(s)-h(r)] \leq 0, \quad s \in[0, r] .
\end{aligned}
$$

Lemma 2.5. Let $\Phi=\Phi^{r}, \Psi=\Psi^{r, R}$ as before. Then there exists $\bar{r}$ such that for each $r>\bar{r}$ there is $R_{r}>r$ such that

$$
\operatorname{sgn}\left(\Phi^{\prime}(r)-\Psi^{\prime}(r)\right)=\operatorname{sgn}\left(R-R_{r}\right) .
$$

Proof. An explicit computation reveals that

$$
\Psi^{\prime}(r)=\left\{\begin{array}{l}
\frac{\mu-a}{r \ln R / r}, \quad n=2, \\
\frac{(n-2)(\mu-a)}{r\left[1-(r / R)^{n-2}\right]}, \quad n>2 .
\end{array}\right.
$$

This and (2.28) show that (2.39) holds with

$$
R_{r}= \begin{cases}r e^{\frac{\mu-a}{r^{\prime}(r)}}, \quad r>\bar{r}=0, & n=2, \\ \frac{r}{\left(1-\frac{(n-2)(\mu-a)}{r \Phi^{\prime}(r)}\right)^{\frac{1}{n-2}}}, & r>\bar{r}=\frac{(n-2)(\mu-a)}{c a}, \quad n>2 .\end{cases}
$$

Remark 2.6. Note that $R_{r}$ is a continuous function of $r \in(\bar{r}, \infty)$ Moreover, $R_{r} \rightarrow$ $+\infty$ for $r \rightarrow+\infty$ and for $r \rightarrow \bar{r}^{+}$. Therefore, there is $r^{*}>0$ such that $R_{r^{*}}$ is minimum.

Let $\vartheta=\vartheta^{r, R}: B_{R} \rightarrow \mathbb{R}$ be defined by

$$
\vartheta^{r, R}:=\chi_{\overline{B_{r}}} \varphi^{r}+\chi_{B_{R} \backslash \overline{B_{r}}} \psi^{r, R} .
$$


We have $\vartheta^{r, R}(x)=\Theta^{r, R}(|x|)$ and $\Theta^{r, R}=\chi_{[0, r]} \Phi^{r}+\chi_{(r, R]} \Psi^{r, R}$. Therefore $\Theta^{\prime}(s)>$ $0, s \in(0, r) \cup(r, R)$ and $\Theta^{\prime}(s)$ is increasing in $(0, r)$ and decreasing in $(r, R)$. In particular, for $0<\delta<\min \{r, R-r\}$,

$$
\Theta(r-\delta)<\Theta(r+\delta)
$$

Lemma 2.7. Assume $r>\bar{r}$ and $R>R_{r}$. Consider $\vartheta$ defined by (2.42) and, fixed $0<\delta<\min \{r, R-r\}$, let $\psi_{\delta}(\cdot)=\Psi_{\delta}(|\cdot|)$ be the solution of

$$
\left\{\begin{array}{l}
\Delta \psi(x)=0, \quad x \in B_{r+\delta} \backslash \bar{B}_{r-\delta}, \\
\psi(x)=\Theta(r-\delta),|x|=r-\delta \\
\psi(x)=\Theta(r+\delta),|x|=r+\delta
\end{array}\right.
$$

Then, $\Psi_{\delta}(s)$ is increasing in $(r-\delta, r+\delta)$ and, if $\delta$ is sufficiently small,

$$
\psi_{\delta}<\vartheta, \quad x \in B_{r+\delta} \backslash \bar{B}_{r-\delta} .
$$

Moreover, there exist $r^{\prime} \in(r, r+\delta)$ and $a^{\prime}<a$ such that

$$
\psi_{\delta} \leq a^{\prime}<a, \quad x \in B_{r^{\prime}} \backslash \bar{B}_{r-\delta} .
$$

Proof. The fact that $\Psi_{\delta}$ is increasing follows from (2.43). From the explicit expression of $\Psi_{\delta}^{\prime}$ one derives that

$$
\Psi_{\delta}^{\prime}(s)=\left(\frac{\Theta^{\prime}\left(r^{-}\right)+\Theta^{\prime}\left(r^{+}\right)}{2 r^{1-n}}+O(\delta)\right) s^{1-n}
$$

and in particular that

$$
\Psi_{\delta}^{\prime}(r-\delta)=\frac{\Theta^{\prime}\left(r^{-}\right)+\Theta^{\prime}\left(r^{+}\right)}{2}\left(\frac{r-\delta}{r}\right)^{1-n}+O(\delta) .
$$

From this and the assumption $R>R_{r}$ that implies $\Theta^{\prime}\left(r^{-}\right)>\Theta^{\prime}\left(r^{+}\right)$, it follows that

$$
\lim _{\delta \rightarrow 0^{+}} \Psi_{\delta}^{\prime}(r-\delta)=\frac{\Theta^{\prime}\left(r^{-}\right)+\Theta^{\prime}\left(r^{+}\right)}{2}<\Theta^{\prime}\left(r^{-}\right) .
$$

Since $\lim _{\delta \rightarrow 0^{+}} \Theta^{\prime}(r-\delta)=\Theta^{\prime}\left(r^{-}\right)$, from (2.49) we have that, for $\delta$ sufficiently small,

$$
\Psi_{\delta}^{\prime}(r-\delta)<\Theta^{\prime}(r-\delta) .
$$

Now, observing that $\Psi_{\delta}(r-\delta)=\Theta(r-\delta)$ and recalling that $\Theta^{\prime}(s)$ is increasing in $(0, r)$ while $\Psi_{\delta}^{\prime}(s)$ is decreasing in $(r-\delta, r+\delta)$, from (2.50) we deduce that

$$
\Psi_{\delta}(s)<\Theta(s) \leq a, \quad s \in(r-\delta, r] .
$$

In particular, $\Psi_{\delta}(r)<a$. This and the maximum principle imply

$$
\Psi_{\delta}(s)<\Theta(s)=\Psi(s), \quad s \in[r, r+\delta),
$$

and (2.45) follows. The last statement of the lemma follows from the fact that $\Psi_{\delta}$ is increasing with $\Psi_{\delta}(r)<a$ and $\Psi_{\delta}(r+\delta)=\Theta(r+\delta)$.

Proposition 2.8. Set $r=r^{*}$, where $r^{*}$ is as in Remark 2.6, and take $R>R_{r^{*}}$. Let $v \in W^{1,2}\left(B_{R}\right)$ satisfy

$$
\begin{gathered}
0 \leq v \leq \mu, \quad x \in B_{R} \\
v \leq a, \quad x \in \bar{B}_{r} .
\end{gathered}
$$


Set

$$
\vartheta_{\delta}=\chi_{B_{R} \backslash\left(B_{r+\delta} \backslash \bar{B}_{r-\delta}\right)} \vartheta+\chi_{B_{r+\delta} \backslash B_{r-\delta}} \psi_{\delta},
$$

where $\psi_{\delta}, \delta>0$ small, as is in Lemma 2.7. Assume

$$
|E|>0, \quad E=\left\{x \in B_{R}: v(x)>\vartheta_{\delta}(x)\right\} .
$$

Then there exists a function $\tilde{v} \in W^{1,2}\left(B_{R}\right)$ such that

$$
\left\{\begin{array}{l}
\tilde{v}=v, \quad x \in \partial B_{R} \\
\tilde{v} \leq \vartheta_{\delta}, \quad x \in B_{R}
\end{array}\right.
$$

and

$$
\mathcal{E}\left(\tilde{v}, B_{R}\right)<\mathcal{E}\left(v, B_{R}\right) .
$$

In particular, (2.57) implies that there are $r^{\prime}>r, a^{\prime}<a$ such that $\tilde{v} \leq a^{\prime}<a$ for $x \in B_{r^{\prime}}$.

Proof. The proof is a direct consequence of Lemmas 2.2, 2.3, and 2.7

We now show that Proposition 2.8 continues to hold when assumption (b) on $W$ is relaxed to (1.7). To see this we set

$$
\omega=\max _{s \in[0, \mu]} W^{\prime}(s)
$$

and change the definition of $\psi$ and $\psi_{\delta}$ by replacing the first equation in (2.8) and (2.44) with $\Delta \psi=-\omega$. This equation and the boundary condition $\Psi(r)=a, \Psi(R)=$ $\mu$ imply

$$
\Psi^{\prime}(r)=-\frac{\omega}{n} r+\frac{\alpha}{r^{n-1}},
$$

where

$$
\alpha=\left\{\begin{array}{l}
\frac{1}{\log \left(\frac{R}{r}\right)}\left[\mu-a+\frac{\omega}{2 n}\left(R^{2}-r^{2}\right)\right], \quad n=2, \\
(n-2) \frac{r^{n-2} R^{n-2}}{R^{n-2}-r^{n-2}}\left[\mu-a+\frac{\omega}{2 n}\left(R^{2}-r^{2}\right)\right], n>2 .
\end{array}\right.
$$

As before we will show that $r$ and $R$ can be chosen so that $\Phi^{\prime}(r)>\Psi^{\prime}(r)$. For fixed $r$ the minimum value of $\Psi^{\prime}(r)$ is obtained for $\alpha=\frac{\omega}{n} R^{n}$. If we substitute this expression of $\alpha$ in (2.60) and (2.61) and set $\rho=R / r$, we get

$$
\begin{gathered}
\Psi^{\prime}(r)=\frac{\omega}{n} r\left(\rho^{n}-1\right), \\
\left\{\begin{array}{l}
\frac{\omega}{n} r^{2}\left(\rho^{2}\left(\log \rho-\frac{1}{2}\right)+\frac{1}{2}\right)=\mu-a, \quad n=2, \\
\frac{\omega}{n} r^{2}\left(\rho^{n}-\frac{n}{2} \rho^{2}+\frac{n-2}{2}\right)=(n-2)(\mu-a), \quad n>2,
\end{array}\right.
\end{gathered}
$$

and finally, after eliminating $r$,

$$
\Psi^{\prime}(r)=\left\{\begin{array}{l}
{\left[\frac{1}{2} \omega(\mu-a)\right]^{1 / 2} \frac{\rho^{2}-1}{\left(\rho^{2}\left(\log \rho-\frac{1}{2}\right)+\frac{1}{2}\right)^{1 / 2}}, n=2} \\
{\left[\frac{n-2}{n} \omega(\mu-a)\right]^{1 / 2} \frac{\rho^{n}-1}{\left(\rho^{n}-\frac{n}{2} \rho^{2}+\frac{n-2}{2}\right)^{1 / 2}}, n>2}
\end{array}\right.
$$


From (2.63) we deduce that $r \rightarrow+\infty$ when $\rho \rightarrow 1^{+}$. This and (2.64) imply

$$
\begin{aligned}
\lim _{r \rightarrow+\infty} \Psi^{\prime}(r) & =(2 \omega(\mu-a))^{1 / 2} \\
& =\left\{\begin{array}{l}
{\left[\frac{1}{2} \omega(\mu-a)\right]^{1 / 2} \lim _{\rho \rightarrow 1^{+}} \frac{\rho^{2}-1}{\left(\rho^{2}\left(\log \rho-\frac{1}{2}\right)+\frac{1}{2}\right)^{1 / 2}}, n=2,} \\
{\left[\frac{n-2}{n} \omega(\mu-a)\right]^{1 / 2} \lim _{\rho \rightarrow 1^{+}} \frac{\rho^{n}-1}{\left(\rho^{n}-\frac{n}{2} \rho^{2}+\frac{n-2}{2}\right)^{1 / 2}}, n>2 .}
\end{array}\right.
\end{aligned}
$$

Then from (2.65) and (2.28) we see that the condition $\omega<c^{2} a^{2} / 2(\mu-a)$ ensures the existence of $r$ and $R$ such that $\Phi^{\prime}(r)>\Psi^{\prime}(r)$. Once this inequality is established, recalling that $V^{\prime}(s) \geq-\omega$, the conclusions of Lemma 2.7 still hold with the same proof when the first equation in (2.44) is replaced by $\Delta \psi=-\omega$. Therefore we conclude that Proposition 2.8 holds.

\section{The Proof of Theorem 1.1}

We first consider the case when $\Omega$ is bounded. Fix a number $R^{*}>R_{r *}$ and take $r=r^{*}$, and $R=R^{*}$ in the definition of the comparison functions $\varphi, \psi, \vartheta, \vartheta_{\delta}$. The assumption that $\Omega$ contains a closed ball of radius $R^{*}$ implies that

$$
\Omega_{R^{*}}=\left\{x \in \Omega: d(x, \partial \Omega)>R^{*}\right\} \neq \emptyset .
$$

The boundedness of $\Omega$ implies that $\Omega_{R^{*}}$ has a finite number of connected components $\Omega_{R^{*}}^{j}, j=1, \ldots, N$. Fix a point $y_{j} \in \Omega_{R^{*}}^{j}$ for each, $j=1, \ldots, N$, and set

$$
\mathcal{A}=\left\{u \in W_{\mu}^{1,2}(\Omega): u(x) \leq a \text { a.e. } x \in \bigcup_{j=1}^{N} \overline{B_{r^{*}}\left(y_{j}\right)}\right\},
$$

where $W_{\mu}^{1,2}(\Omega)$ denotes the set of the functions in $W^{1,2}(\Omega)$ with trace $\mu$ on $\partial \Omega$.

Proposition 3.1. Assume $u \in \mathcal{A}$. Then there exist $a^{\prime}, \delta^{\prime}>0$ and $v \in \mathcal{A}$ such that

$$
\begin{gathered}
0 \leq v \leq \mu \quad \text { a.e. } x \in \Omega \\
v \leq a^{\prime}<a \quad \text { a.e. } x \in \Omega_{R^{*}}+B_{r^{*}}+B_{\delta^{\prime}},
\end{gathered}
$$

and

$$
\mathcal{E}(v, \Omega) \leq \mathcal{E}(u, \Omega)
$$

Proof. 1. By Lemma 2.1 we can restrict ourselves to the subset of $\mathcal{A}$ of the functions verifying (3.3).

2. Since $R^{*}>R_{r^{*}}$, from Proposition 2.8 we have the existence of $a^{\prime}, \delta^{\prime}>0$ such that, given $z \in \Omega_{R^{*}}$ and $w \in \mathcal{A}$, the condition

$$
w(x) \leq a \quad \text { a.e. } x \in \overline{B_{r^{*}}(z)}
$$

implies the existence of a function $\tilde{w}$ satisfying

$$
\tilde{w}(x) \leq a^{\prime}<a \quad \text { a.e. } x \in B_{r^{*}}(z)+B_{\delta^{\prime}},
$$

and $\mathcal{E}(\tilde{w}, \Omega) \leq \mathcal{E}(w, \Omega)$.

3. For each fixed $y \in \Omega_{R^{*}}$ there exist $1 \leq j \leq N$ and points $z_{h} \in \Omega_{R}^{j}, h=$ $0, \ldots, M$, such that

$$
\begin{gathered}
z_{0}=y_{j}, \quad z_{M}=y, \\
\overline{B_{r^{*}}\left(z_{h}\right)} \subset B_{r^{*}}\left(z_{h-1}\right)+B_{\delta^{\prime}}, h=1, \ldots, M .
\end{gathered}
$$


Since $u \in \mathcal{A}, w=u$ and $z=y_{j}$ satisfy (3.6). This, together with steps 2 and 3 and an induction argument, prove the existence of $v \in \mathcal{A}$ also satisfying (3.4) and (3.5). This concludes the proof.

Corollary 3.2. The function $v$ in Proposition 3.1 can be chosen such that

$$
v(x) \leq K e^{-k d(x, \partial \Omega)}, x \in \Omega,
$$

where $k=h\left(r^{*}\right)$, $h$ the function in Lemma 2.4, and $K=e^{h\left(r^{*}\right) R^{*}} \mu$.

Proof. First consider $x \in \Omega_{R^{*}}$ and set $\rho(x)=d\left(x, \partial\left(\Omega_{R^{*}}+B_{r^{*}}\right)\right)$. Then $B_{\rho(x)}(x) \subset$ $\Omega_{R^{*}}+B_{r^{*}}$, and from (3.4) we have that

$$
v<a \quad \text { a.e. } y \in B_{\rho(x)}(x) .
$$

Therefore Lemma 2.2 and (2.29) imply that

$$
v(x) \leq e^{-h(\rho(x)) \rho(x)} a \leq e^{-h\left(r^{*}\right) \rho(x)} a \quad \text { a.e. } x \in \Omega_{R^{*}},
$$

where we have also used the monotonicity of $h$ and $\rho(x) \geq r^{*}$. Now, observing that, for $x \in \Omega_{R^{*}}, d(x, \partial \Omega) \leq \rho(x)+R^{*}$, from (3.12) we deduce that

$$
v(x) \leq e^{h\left(r^{*}\right)\left(R^{*}-d(x, \partial \Omega)\right)} a \quad \text { a.e. } x \in \Omega_{R^{*}} .
$$

If $x \in \Omega \backslash \Omega_{R^{*}}$ we trivially have

$$
v(x) \leq \mu \leq e^{h\left(r^{*}\right)\left(R^{*}-d(x, \partial \Omega)\right)} \mu \quad \text { a.e. } x \in \Omega \backslash \Omega_{R^{*}} .
$$

Thus, estimate (3.10) holds for any $x \in \Omega$ with $k=h\left(r^{*}\right)$ and $K=\mu e^{h\left(r^{*}\right) R^{*}}$.

Theorem 3.3. Assume that $\Omega$ is a Lipschitz bounded domain and assume that $W$ satisfies (a)-(c). Then all the statements of Theorem 1.1 hold true.

Proof. Suppose that $\Omega$ contains a closed ball of radius $R^{*}$. Then there exists $v \in \mathcal{A}$ such that

$$
\mathcal{E}(v, \Omega)=\min _{u \in \mathcal{A}} \mathcal{E}(u, \Omega)<+\infty .
$$

The condition $\overline{B_{R^{*}}(x)} \subset \Omega$ for some $x \in \Omega$ implies $\Omega_{R^{*}} \neq \emptyset$, and therefore the set $\mathcal{A}$ is nonempty. For instance the function $u_{0}$ defined by

$$
u_{0}(x)=\min \left\{1, \frac{d\left(x, \bigcup_{j=1}^{N} B_{r^{*}}\left(y_{j}\right)\right)}{R^{*}-r^{*}}\right\} \mu,
$$

belongs to $\mathcal{A}$ and satisfies $\mathcal{E}_{0}=\mathcal{E}\left(u_{0}, \Omega\right)<+\infty$. Let $\left\{v_{h}\right\}_{h=1}^{\infty} \subset \mathcal{A}$, a minimizing sequence. We can assume that

$$
\mathcal{E}\left(v_{h}, \Omega\right) \leq \mathcal{E}_{0},
$$

and by Proposition 3.1 and Corollary 3.2 we can also assume that $v_{h}$ satisfies (3.3), (3.4) and the exponential estimate (3.10). From (3.16) it follows that $\int_{\Omega}\left|\nabla v_{h}\right|^{2} \leq 2 \mathcal{E}_{0}$, which implies the existence of a subsequence $v_{h_{j}}$ that converges weakly and pointwise to a function $v \in \mathcal{A}$ that satisfies (3.3), (3.4) and (3.10). By weak convergence we have

$$
\int_{\Omega}|\nabla v|^{2} \leq \liminf _{j \rightarrow+\infty} \int_{\Omega}\left|\nabla v_{h_{j}}\right|^{2}
$$

and, recalling that $V \geq 0$, we can apply Fatou's Lemma to obtain

$$
\int_{\Omega} V(v) \leq \liminf _{j \rightarrow+\infty} \int_{\Omega} V\left(v_{h_{j}}\right) .
$$


From (3.17) and (3.18) we have that $v$ satisfies (3.15). Moreover, from (3.4) we have in particular that

$$
v(x) \leq a^{\prime}<a \quad \text { a.e. } x \in B_{r^{*}}\left(y_{j}\right)+B_{\delta^{\prime}}, j=1, \ldots, N ;
$$

that is, $v$ stays away from the constraint imposed by membership in $\mathcal{A}$. It follows that $v$ is a free minimizer and therefore a weak solution of (2.2). Since $0 \leq v \leq \mu$ and $V^{\prime}(s)$ is bounded on $[0, \mu]$, we have

$$
\|v\|_{L^{\infty}(\Omega)}+\left\|V^{\prime}(v)\right\|_{L^{\infty}(\Omega)} \leq \mu+\left.\max V^{\prime}\right|_{[0, \mu]} .
$$

Let $B_{\rho}$ be a ball such that $\overline{B_{\rho}} \subset \Omega$. From this, (3.20) and the interior elliptic estimate, assuming $p>n$, and also using the Sobolev inequality $\|v\|_{C^{1, \alpha}\left(\overline{B_{\rho}}\right)} \leq$ $C\|v\|_{W^{2, p}\left(B_{\rho}\right)}, \alpha \in(0,1)$, we get

$$
\|v\|_{C^{1, \alpha}\left(\overline{B_{\rho}}\right)} \leq C\|v\|_{W^{2, p}\left(B_{\rho}\right)} \leq C_{1}\left(\|v\|_{L^{\infty}(\Omega)}+\left\|V^{\prime}(v)\right\|_{L^{\infty}(\Omega)}\right) \leq C_{2},
$$

where $C_{i}, i=1,2$, depend only on $n, \rho$ and on the distance $d\left(B_{\rho}, \partial \Omega\right)$. Since $V^{\prime \prime}$ is bounded in $[0, \mu]$ and (3.21) implies $\|\nabla v\|_{L^{\infty}\left(\overline{B_{\rho}}\right)} \leq C_{2}$, we can differentiate (2.2) and apply (3.21) to $\nabla v$ to conclude that for each ball $B_{\rho}$ such that $\overline{B_{\rho}} \subset \Omega$ we have

$$
\|v\|_{C^{2, \alpha}\left(\overline{B_{\rho}}\right)} \leq C,
$$

where the constant $C$ depends only on $n, \rho$ and on the distance $d\left(B_{\rho}, \partial \Omega\right)$. This shows that $v$ is a classical solution of (2.2). The assumption that $\Omega$ is Lipschitz implies that $v$ can be extended to $\bar{\Omega}$ as a continuous function that vanishes on $\partial \Omega$ (see Theorem 8.30 in [8]). It remains to prove that $v$ satisfies (3.3) with the sign of strict inequality. This follows from $0 \leq v \leq \mu, V^{\prime}(v) \geq 0$ and the strong maximum principle. The equivalence between (1.1) and (2.2) concludes the proof.

We now remove the assumption that $\Omega$ is bounded and prove Theorem 1.1. We assume that $0 \in \Omega$ and let $\Omega_{1}$ be the connected component of $\Omega \cap B_{1}(0)$ that contains the origin. For $h>1$ we define $\Omega_{h}$ as the connected component of $\Omega \cap B_{h}(0)$ that contains $\Omega_{h-1}$. Then

$$
\Omega=\bigcup_{h=1}^{\infty} \Omega_{h}
$$

Moreover, we have

$$
\begin{aligned}
\partial \Omega \cap \partial \Omega_{h} & \subset \partial \Omega \cap \partial \Omega_{h+1}, \\
\partial \Omega & =\bigcup_{h=1}^{\infty} \partial \Omega \cap \partial \Omega_{h} .
\end{aligned}
$$

Let $B_{\rho_{j}}\left(x_{j}\right), j \in \mathbb{N}$, be a sequence of balls such that

$$
\begin{array}{r}
\overline{B_{\rho_{j}}\left(x_{j}\right)} \subset \Omega, \\
\Omega=\bigcup_{j=1}^{\infty} B_{\rho_{j}}\left(x_{j}\right) .
\end{array}
$$

Then, for each $j \in \mathbb{N}$, there is $h_{j}$ such that $h \geq h_{j}$ implies

$$
\overline{B_{\rho_{j}}\left(x_{j}\right)} \subset \Omega_{h_{j}}, 0<d\left(B_{\rho_{j}}\left(x_{j}\right), \partial \Omega_{h_{j}}\right) \leq d\left(B_{\rho_{j}}\left(x_{j}\right), \partial \Omega_{h}\right) .
$$


From Theorem 3.3 we obtain a sequence $v_{h}, h \in \mathbb{N}$, of solutions of (2.2) in $\Omega_{h}$. From (3.22) and (3.26) it follows that for each $j \in \mathbb{N}$ there is a constant $C_{j}$ such that

$$
\left\|v_{h}\right\|_{C^{2, \alpha}\left(\overline{B_{\rho_{j}}\left(x_{j}\right)}\right)} \leq C_{j}, h \geq h_{j} .
$$

Therefore the Ascoli-Arzelá Theorem implies that for each $j \in \mathbb{N}$ there is a subsequence $\left\{v_{h_{k}^{j}}\right\}$ of $\left\{v_{h}\right\}$ that converges in $C^{2}\left(\left(\overline{B_{\rho_{j}}\left(x_{j}\right)}\right)\right.$. We can assume that $\left\{v_{h_{k}^{j+1}}\right\}$ is a subsequence of $\left\{v_{h_{k}^{j}}\right\}$, and therefore by Cantor diagonalization we have that the sequence $w_{k}=v_{h_{k}^{k}}$ converges in $C^{2}\left(\left(\overline{B_{\rho_{j}}\left(x_{j}\right)}\right)\right.$ for each $j \in \mathbb{N}$ and pointwise to a $C^{2}$ function $v$ that is a classical solution of (2.2) and satisfies (3.3), (3.4) and (3.10). For each $k \in \mathbb{N}$ we have $w_{k} \in W_{0}^{1,2}\left(\Omega_{h_{k}^{k}}\right)$. Finally a further application of the strong maximum principle establishes the strict inequality in (3.3). The equivalence of (2.2) and (1.1) concludes the proof.

Proof of Theorem 1.6. By examining the proof of Theorem 1.1 we see that the only thing we need to check is that, under the assumptions (1.10), (1.11), and (1.12), there exist sequences $\lambda_{j}, r_{j}, R_{j}, j \in \mathbb{N}$, that converge to zero and

$$
\varphi_{j}^{\prime}\left(r_{j}\right)>\psi_{j}^{\prime}\left(r_{j}\right), \quad j \in \mathbb{N},
$$

where $\varphi_{j}, \psi_{j}$ are the solutions of problems (2.7) and (2.8) with $a=a_{j}, \mu=\mu_{j}$, $c=\lambda_{j}^{1 / 2} c_{j}, r=r_{j}, R=R_{j}$. Indeed, once this inequality is established, given $\lambda>0$ and a domain $\Omega$, there exists $i=i(\lambda, \Omega)$ such that $j>i$ implies

$$
\lambda_{j}<\lambda, \quad R_{j}<R_{\Omega}=\sup \left\{R: B_{R}(x) \subset \Omega\right\} .
$$

This implies that the sufficient condition required in Theorem 1.1 for the existence of a positive solution of (1.9) in the interval $\left[0, \mu_{j}\right]$ is satisfied for all $j>i$. From Theorem 1.1 it follows that, up to subsequences,

$$
\mu_{j-1} \leq \mu_{j}-a_{j}<\max u_{j}<\mu_{j}, \quad j>i+1,
$$

and then $u_{j} \neq u_{h}$, for $j \neq h$. It remains to show the existence of the sequences $\lambda_{j}, r_{j}, R_{j}, j \in \mathbb{N}$. To this end, let $\Phi: B_{1} \times(0,+\infty) \rightarrow \mathbb{R}, \Phi:=\Phi(x, \gamma)$, be the solution of the problem

$$
\begin{cases}\Delta \Phi=\gamma^{2} \Phi, & x \in B_{1} \\ \Phi=1, & x \in \partial B_{1}\end{cases}
$$

From (2.28) with $\gamma=c r$ one derives that

$$
\lim _{\gamma \rightarrow+\infty} \Phi^{\prime}(1, \gamma)=+\infty
$$

If we set $\gamma_{j}^{2}=\lambda_{j} c_{j}^{2} r_{j}^{2}$ we have the identity $\varphi_{j}(|x|)=a_{j} \Phi\left(\frac{|x|}{r_{j}}, \gamma_{j}\right)$ which implies $\varphi_{j}^{\prime}\left(r_{j}\right)=\frac{a_{j}}{r_{j}} \Phi^{\prime}\left(1, \gamma_{j}\right)$. From this and (2.40) with $a, \mu, r, R$ as before, we see that (3.28) is equivalent to

$$
\Phi^{\prime}\left(1, \gamma_{j}\right)>\frac{\mu_{j}-a_{j}}{a_{j}} \frac{n-2}{1-\left(r_{j} / R_{j}\right)^{n-2}} .
$$

We fix a number $\eta \in(0,1)$ and set $R_{j}=\frac{1}{\eta} r_{j}, j \in \mathbb{N}$. Then, (1.12) and (3.32) imply that there is a $\bar{\gamma}>0$ such that

$$
\Phi^{\prime}(1, \bar{\gamma})>C \frac{n-2}{1-\eta^{n-2}} \geq \frac{\mu_{j}-a_{j}}{a_{j}} \cdot \frac{n-2}{1-\left(r_{j} / R_{j}\right)^{n-2}}, \quad j \in \mathbb{N} .
$$


Fix

$$
\bar{\gamma}=\gamma_{j}=\lambda_{j}^{1 / 2} c_{j} r_{j}, \quad j \in \mathbb{N} .
$$

By assumption, $\lim _{j \rightarrow+\infty} c_{j}=+\infty$, therefore we can take for $\lambda_{j}, r_{j}, j \in \mathbb{N}$, any pair of sequences that converge to zero and satisfy (3.34).

\section{The miXed Dirichlet-Neumann Boundary CONDition}

In this section we study problem (1.13) and prove Theorem 1.9. The proof is a slight variation of the proof of Theorem 1.1. and it is based on simple extensions of the comparison lemmas, Lemmas 2.2 and 2.3, that allow us to cover the case when the ball $B_{R^{*}}(x)$ intersects $\Gamma_{N}$. We rewrite problem (1.13) in the equivalent form:

$$
\begin{cases}\Delta v=V^{\prime}(v), & x \in \Omega, \\ v=\mu, & x \in \Gamma_{D}, \\ \frac{\partial v}{\partial \nu}=0, & x \in \Gamma_{N} .\end{cases}
$$

For each $x \in \bar{\Omega}$ and $\rho>0$, let $\mathcal{B}_{\rho}(x)$ and $\partial_{\Omega} \mathcal{B}_{\rho}(x)$ be the sets defined in section 1. By a translation we can always reduce to the case $x=0$; therefore we assume $x=0$ and set $\mathcal{B}_{\rho}=\mathcal{B}_{\rho}(0), \partial_{\Omega} \mathcal{B}_{\rho}=\partial_{\Omega} \mathcal{B}_{\rho}(0)$.

Lemma 4.1. Let $a, c$ be as in (1.6), $\varphi$ be the solution of (2.7) and let $v \in W^{1,2}\left(\mathcal{B}_{r}\right)$ satisfy

$$
\begin{gathered}
0 \leq v \leq a, x \in \overline{\mathcal{B}_{r}} \\
|E|>0, \quad \text { where } \quad E=\left\{x \in \mathcal{B}_{r}: v(x)>\varphi(x)\right\} .
\end{gathered}
$$

Moreover, we assume that

$$
x \cdot \nu(x) \geq 0, \quad x \in \partial_{\Omega} \mathcal{B}_{r} .
$$

Then there is a function $\tilde{v} \in W^{1,2}\left(\mathcal{B}_{r}\right)$ verifying

$$
\left\{\begin{array}{l}
\tilde{v}=v, \quad x \in \mathcal{B}_{r} \backslash E \\
\tilde{v} \leq \varphi, \quad x \in E
\end{array}\right.
$$

and

$$
\mathcal{E}\left(\tilde{v}, \mathcal{B}_{r}\right)<\mathcal{E}\left(v, \mathcal{B}_{r}\right)
$$

Proof. Let $\eta \in W^{1,2}\left(\mathcal{B}_{r}\right)$ be a function vanishing, in the trace sense, on $\partial \mathcal{B}_{r} \backslash \partial_{\Omega} \mathcal{B}_{r}$. Then, integrating (2.7) on $\mathcal{B}_{r}$ we have

$$
\int_{\mathcal{B}_{r}}\left(\nabla \varphi \nabla \eta+c^{2} \varphi \eta\right) d x-\int_{\partial_{\Omega} \mathcal{B}_{r}} \frac{\partial \varphi}{\partial \nu} \eta d \Gamma=0 .
$$

In particular, if we take

$$
\eta(x)= \begin{cases}0 & \text { for } x \in \mathcal{B}_{r} \backslash E \\ v(x)-\varphi(x) & \text { for } x \in E\end{cases}
$$

from (4.7) it follows that

$$
\int_{E}\left\{\nabla \varphi(\nabla v-\nabla \varphi)+c^{2} \varphi(v-\varphi)\right\} d x-\int_{\partial_{\Omega} \mathcal{B}_{r} \cap E} \frac{\partial \varphi}{\partial \nu}(v-\varphi) d \Gamma=0 .
$$


Then, proceeding as in the proof of Lemma 2.2 we obtain, instead of (2.18), $(4.10)$

$$
\begin{aligned}
\mathcal{E}\left(v, \mathcal{B}_{r}\right)- & \mathcal{E}\left(\tilde{v}, \mathcal{B}_{r}\right) \\
& =\frac{1}{2} \int_{E}|\nabla v-\nabla \varphi|^{2} d x+\int_{E}\left[V(v)-U_{\varphi}(v)\right] d x+\int_{\partial_{\Omega} \mathcal{B}_{r} \cap E} \frac{\partial \varphi}{\partial \nu}(v-\varphi) d \Gamma .
\end{aligned}
$$

From this inequality (4.6) follows by the same argument as in the proof of Lemma 2.2. provided we show that the boundary integral on the right hand side of (4.10) is nonnegative. This follows from assumption (4.4) recalling that $\Phi^{\prime}(r)>0$, and so

$$
\frac{\partial \varphi}{\partial \nu}(x)=\Phi^{\prime}(r) \frac{x}{|x|} \cdot \nu(x) \geq 0, \quad x \in \partial_{\Omega} \mathcal{B}_{r}
$$

The following extensions of Lemma 2.3 and Proposition 2.8 are proved in a similar way.

Lemma 4.2. Let $\psi$ be the solution of (2.8) and assume that $v \in W^{1,2}\left(\mathcal{B}_{R} \backslash \overline{\mathcal{B}}_{r}\right)$ satisfies

$$
\begin{gathered}
0 \leq v \leq \mu, x \in \mathcal{B}_{R} \backslash \overline{\mathcal{B}}_{r}, \\
v \leq \psi, x \in \partial\left(\mathcal{B}_{R} \backslash \overline{\mathcal{B}}_{r}\right) \backslash \partial_{\Omega} \mathcal{B}_{R}, \\
|E|>0, \quad \text { where } \quad E=\left\{x \in \mathcal{B}_{R} \backslash \overline{\mathcal{B}}_{r}: v(x)>\psi(x)\right\} .
\end{gathered}
$$

Then, provided

$$
x \cdot \nu(x) \geq 0, \quad x \in \partial_{\Omega} \mathcal{B}_{R},
$$

there is a function $\tilde{v} \in W^{1,2}\left(\mathcal{B}_{R} \backslash \overline{\mathcal{B}}_{r}\right)$ such that

$$
\left\{\begin{array}{l}
\tilde{v}=v, \quad x \in\left(\mathcal{B}_{R} \backslash \overline{\mathcal{B}}_{r}\right) \backslash E, \\
\tilde{v} \leq \psi, \quad x \in E
\end{array}\right.
$$

and

$$
\mathcal{E}\left(\tilde{v}, \mathcal{B}_{R} \backslash \overline{\mathcal{B}}_{r}\right) \leq \mathcal{E}\left(v, \mathcal{B}_{R} \backslash \overline{\mathcal{B}}_{r}\right)
$$

Proposition 4.3. Let $a, c$ be as in (1.6). Set $r=r^{*}$, where $r^{*}$ is as in Remark 2.6, and take $R>R_{r^{*}}$. Let $v \in W^{1,2}\left(\mathcal{B}_{R}\right)$ satisfy

$$
\begin{gathered}
0 \leq v \leq \mu, x \in \mathcal{B}_{R}, \\
v \leq a, \quad x \in \overline{\mathcal{B}_{r}}
\end{gathered}
$$

and

$$
\begin{aligned}
& |E|>0, \quad \text { where } \quad E=\left\{x \in \mathcal{B}_{R}: v(x)>\vartheta_{\delta}(x)\right\} \quad \text { with } \\
& \vartheta_{\delta}=\chi_{B_{R} \backslash\left(B_{r+\delta} \backslash \bar{B}_{r-\delta}\right)} \vartheta+\chi_{B_{r+\delta} \backslash B_{r-\delta}} \psi_{\delta} .
\end{aligned}
$$

Then, provided that assumption (4.14) holds, there exists a function $\tilde{v} \in W^{1,2}\left(\mathcal{B}_{R}\right)$ such that

$$
\left\{\begin{array}{l}
\tilde{v}=v, \quad x \in \partial \mathcal{B}_{R} \backslash \partial_{\Omega} \mathcal{B}_{R} \\
\tilde{v} \leq \vartheta_{\delta}, \quad x \in \mathcal{B}_{R}
\end{array}\right.
$$

and

$$
\mathcal{E}\left(\tilde{v}, \mathcal{B}_{R}\right)<\mathcal{E}\left(v, \mathcal{B}_{R}\right)
$$

In particular, (4.20) implies that there is $r^{\prime}>r$ such that $\tilde{v}<a^{\prime}<a$ for $x \in \mathcal{B}_{r^{\prime}}$. 
Fix a number $R^{*}>R_{r^{*}}$ and take $r=r^{*}, R=R^{*}$ in the definition of the functions $\varphi, \psi, \psi_{\delta}, \ldots$. Proceeding as in the proof of Theorem 1.1 we first consider the case of $\Omega$ bounded and observe that the assumption $\overline{\mathcal{B}_{R^{*}}(x)} \cap \bar{\Gamma}_{D}=\emptyset$ implies that the set $\Omega_{R^{*}}^{\mathcal{N}} \subset \Omega$ defined by

$$
\Omega_{R^{*}}^{\mathcal{N}}=\left\{x \in \Omega: \overline{\mathcal{B}_{R^{*}}(x)} \cap \bar{\Gamma}_{D}=\emptyset\right\}
$$

is open and nonempty. Let $\Omega_{R^{*}}^{\mathcal{N}, j}, j=1, \ldots, N$, be the connected components of $\Omega_{R^{*}}^{\mathcal{N}}$. Fix a point $y_{j} \in \Omega_{R^{*}}^{\mathcal{N}, j}, j=1, \ldots, N$, and set

$$
\mathcal{A}^{\mathcal{N}}=\left\{u \in W_{\mu, \Gamma_{D}}^{1,2}(\Omega): u(x) \leq a \text { a.e. } x \in \bigcup_{j=1}^{N} \overline{\mathcal{B}_{r^{*}}\left(y_{j}\right)}\right\}
$$

where $W_{\mu, \Gamma_{D}}^{1,2}(\Omega)$ denotes the set of the functions of $W^{1,2}(\Omega)$ with trace $\mu$ on $\Gamma_{D}$.

Proposition 4.4. Let $u$ be in $\mathcal{A}^{\mathcal{N}}$. Then there exist $a^{\prime}, \delta^{\prime}>0$ and $v \in \mathcal{A}^{\mathcal{N}}$ such that

$$
\begin{gathered}
0 \leq v \leq \mu \quad \text { a.e. } x \in \Omega, \\
v \leq a^{\prime}<a \quad \text { a.e. } \quad x \in \bigcup_{y \in \Omega_{R^{*}}^{\mathcal{N}}} \mathcal{B}_{r^{*}+\delta^{\prime}}(y)
\end{gathered}
$$

and

$$
\mathcal{E}(v, \Omega) \leq \mathcal{E}(u, \Omega) .
$$

Proof. The proof is analogous to the proof of Proposition 3.1 and is omitted.

Corollary 4.5. The function $v$ in Proposition 4.4 can be chosen such that

$$
v(x) \leq K e^{-k \mathcal{G}\left(x, \Gamma_{D}\right)}, x \in \Omega,
$$

where $k=h\left(r^{*}\right), h$ is the function in Lemma 2.4, and $K=e^{h\left(r^{*}\right) R^{*}} \mu$.

Proof. First consider the case $x \in \Omega_{R^{*}}^{\mathcal{N}}$ and let

$$
\rho(x)=\sup \left\{\rho>0: \mathcal{B}_{\rho}(x) \subset \bigcup_{y \in \Omega_{R^{\mathcal{N}}}} \mathcal{B}_{r^{*}}(y)\right\} .
$$

Then, as in the proof of Corollary 3.2, we get

$$
v(x) \leq e^{-h\left(r^{*}\right) \rho(x)} a \quad \text { a.e. } x \in \Omega_{R^{*}}^{\mathcal{N}} .
$$

Given $x \in \Omega_{R^{*}}^{\mathcal{N}}$, let

$$
\mathrm{r}(x)=\sup \left\{\mathrm{r}>0: \mathcal{B}_{\mathrm{r}}(x) \subset \Omega_{R^{*}}^{\mathcal{N}}\right\}
$$

and note that

$$
r(x) \leq \rho(x) .
$$

Define $\partial_{B} \mathcal{B}_{r}(x):=\partial \mathcal{B}_{r}(x) \cap \partial B_{r}(x)$.

The maximality of $\mathrm{r}(x)$ implies the existence of $y \in \overline{\partial \Omega_{R^{*}}^{\mathcal{N}} \backslash \partial \Omega}$ such that

(i) $y \in \partial_{B} \mathcal{B}_{\mathrm{r}(x)}(x)$,

(ii) $\partial_{B} \mathcal{B}_{R^{*}}(y) \cap \bar{\Gamma}_{D} \neq \emptyset$. 
From (i) and (4.31) we have

$$
|y-x|=r(x) \leq \rho(x),
$$

and from (ii) there exists $z \in \bar{\Gamma}_{D}$ such that

$$
d\left(y, \Gamma_{D}\right)=|z-y|=R^{*} .
$$

Therefore, also using the fact that the convexity of $\Gamma_{N}$ implies that both $\mathcal{B}_{\mathrm{r}(x)}(x)$ and $\mathcal{B}_{R^{*}}(y)$ are convex sets, we conclude

$$
\mathcal{G}\left(x, \Gamma_{D}\right) \leq|y-x|+d\left(y, \Gamma_{D}\right)=\rho(x)+R^{*} .
$$

It follows that

$$
v(x) \leq e^{-h\left(r^{*}\right)\left(\mathcal{G}\left(x, \Gamma_{D}\right)-R^{*}\right)} a \quad \text { a.e. } x \in \Omega_{R^{*}}^{\mathcal{N}} .
$$

Now assume $x \in \Omega \backslash \Omega_{R^{*}}^{\mathcal{N}}$ and let $\rho(x)=\sup \left\{\rho: \mathcal{B}_{\rho}(x) \cap \Gamma_{D}=\emptyset\right\}$. Then, by definition, there exists $y \in \bar{\Gamma}_{D}$ such that $|y-x| \leq \rho(x) \leq R^{*}$, and it follows that

$$
\mathcal{G}\left(x, \Gamma_{D}\right) \leq|y-x| \leq R^{*} .
$$

This implies

$$
v(x) \leq \mu \leq e^{h\left(r^{*}\right)\left(R^{*}-\mathcal{G}\left(x, \Gamma_{D}\right)\right)} \mu \quad \text { a.e. } x \in \Omega \backslash \Omega_{R^{*}}^{\mathcal{N}}
$$

and concludes the proof.

Proof of Theorem 1.9. As in the proof of Theorem 1.1 we first assume $\Omega$ bounded and find a minimizer $v \in \mathcal{A}^{\mathcal{N}}$ of $\mathcal{E}(u, \Omega)$. By Proposition 4.4 and Corollary $4.5 v$ is a weak solution of (4.1) and the estimate (4.27) holds. By interior elliptic regularity $v \in C^{2, \alpha}\left(B_{\rho}\right), \alpha \in(0,1)$, for all balls $B_{\rho} \Subset \Omega$. Then by applying the same limit procedure as in the Dirichlet case, we obtain a solution $v \in C^{2}(\Omega)$ of (4.1) for $\Omega$ unbounded. The assumption that $\Omega$ is $C^{2}$ implies that $v$ can be extended to $\bar{\Omega}$ as a continuous function satisfying the Dirichlet condition on $\Gamma_{D}$. By a result in [13] (see sec. 6 in [13]) $v$ satisfies the Neumann boundary condition on $\Gamma_{N}$.

For the analysis of the regularity of the solution of problem (1.13) when $\bar{\Gamma}_{D} \cap$ $\bar{\Gamma}_{N} \neq \emptyset$, we refer to $[12$ and to the references therein.

\section{ON THE MIXED PROBLEM IN MORE GENERAL DOMAINS}

We consider an open connected set $\Omega$ and $\Gamma_{D}, \Gamma_{N} \subset \partial \Omega$ that satisfy

(H1) $\Omega$ is $C^{2}$ and $\Gamma_{D}, \Gamma_{N}=\partial \Omega \backslash \bar{\Gamma}_{D}$ are open $(n-1)$-dimensional smooth submanifolds of $\partial \Omega$

and the assumptions (H2), (H3) below.

Let $\Sigma$ be defined by

$\Sigma=\left\{x \in \bar{\Omega}: d\left(x, \Gamma_{D}\right)=R^{*}, \overline{\mathcal{B}}_{R^{*}}(x) \cap \bar{\Gamma}_{D} \neq \emptyset,(y-x) \cdot \nu(y) \geq 0, \forall y \in \partial_{\Omega} \mathcal{B}_{R^{*}}(x)\right\}$.

Set

$$
\omega=\bigcup_{x \in \Sigma} \mathcal{B}_{r^{*}}(x)
$$

and let $\Omega_{D} \subset \Omega$ be the union of the connected components $\Omega_{j}$ of $\Omega \backslash \bar{\omega}$ that touch $\Gamma_{D}$ in the sense that

$$
\bar{\Omega}_{j} \cap \Gamma_{D} \neq \emptyset .
$$




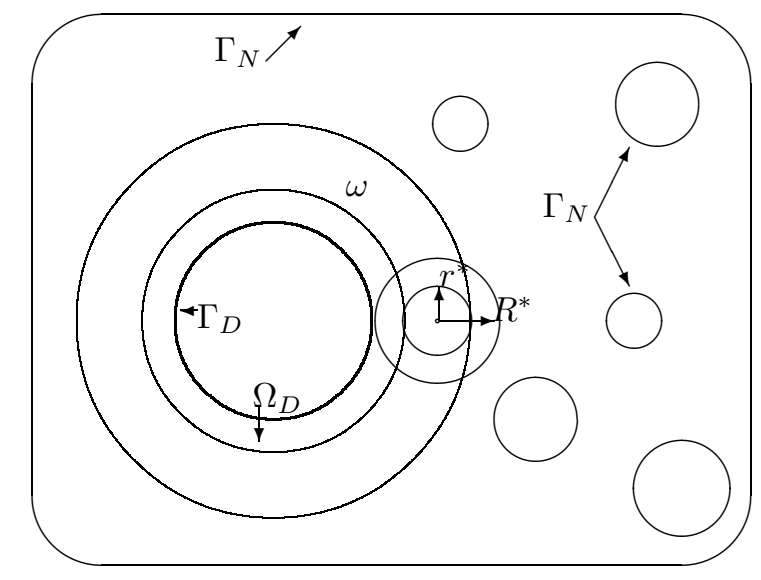

Figure 2. $\Omega, \Gamma_{D}, \Gamma_{N}$ that satisfy the assumptions in Theorem 5.2

Set

$$
\Omega_{N}=\Omega \backslash \bar{\Omega}_{D}
$$

Then:

(H2) $\Omega_{D} \subset\left\{x \in \mathbb{R}^{n}: d\left(x, \Gamma_{D}\right)<R^{*}\right\}$;

(H3) there exist $\rho, \rho_{0}$ with $0<\rho_{0}<\rho$ such that for each $x \in \overline{\Omega_{N} \backslash \omega}$ there is $x_{0} \in \Omega_{N}$ that satisfies

$$
\left\{\begin{array}{l}
\left|x-x_{0}\right| \leq \rho_{0}, \mathcal{B}_{\rho}\left(x_{0}\right) \subset \Omega_{N} \\
\left(y-x_{0}\right) \cdot \nu(y) \geq 0, \quad y \in \partial_{\Omega} \mathcal{B}_{\rho}\left(x_{0}\right) .
\end{array}\right.
$$

Remark 5.1. Condition (H2) says that the set $\omega$ cuts away from the rest of $\Omega$ a neighborhood of $\Gamma_{D}$ of radius $R^{*}$. If $\tilde{\Omega}, \tilde{\Gamma}_{D}$, and $\tilde{\Gamma}_{N}$ satisfy $(\mathbf{H 1})$ and $\tilde{\Omega}$ is bounded or $\partial \tilde{\Omega}$ has bounded curvatures, then $\Omega=\lambda \tilde{\Omega}, \lambda \tilde{\Gamma}_{D}$ and $\lambda \tilde{\Gamma}_{N}$ satisfy conditions (H1)-(H3) for $\lambda$ sufficiently large.

Theorem 5.2. Let $\Omega, \Gamma_{D}$ and $\Gamma_{N}$ satisfy (H1)-(H3) and let $W$ satisfy (a)-(c). Then problem (1.13) has a positive solution $u \in C^{2}(\Omega)$ such that

$$
0<\mu-u(x)<K e^{-k \mathcal{G}\left(x, \Gamma_{D}\right)}, \quad x \in \bar{\Omega},
$$

for some positive constants $K, k$ depending on $W, n, \rho_{0}, \rho$.

Proof. We only discuss the case of $\Omega$ bounded. As before we transform (1.13) into the equivalent problem for $v=\mu-u$. We choose $v$ to be a minimizer of $\mathcal{E}(v, \Omega)$ on the set $\mathcal{A}$ of admissible functions $v \in W_{\mu, \Gamma_{D}}^{1,2}(\Omega)$ defined by

$$
v(x) \leq a e^{-k \mathcal{G}\left(x, \Omega_{D}\right)}, \quad x \in \bar{\Omega}_{N},
$$

for a fixed number $k>0$ satisfying

$$
k<\min \left\{-\frac{1}{\rho+\rho_{0}} \ln \frac{\tilde{\varphi}\left(\rho_{0}\right)}{\tilde{\varphi}(\rho)},-\frac{1}{R^{*}+r^{*}} \ln \frac{a^{\prime}}{a}\right\},
$$


where $\tilde{\varphi}$ is the solution of (2.7) in $B_{\rho}$ and $a^{\prime}<a$ is the constant appearing in Propositions 3.1 and 4.4. Note that the ratio $\tilde{\varphi}\left(\rho_{0}\right) / \tilde{\varphi}(\rho)$ is independent of the boundary datum $\tilde{\varphi}(\rho)$.

Assume first that $x \in \bar{\omega}$. Then, as in the proof of Proposition 3.1 and 4.4, we deduce from Proposition 4.3 that

$$
v(x) \leq a^{\prime}
$$

For $x \in \bar{\omega}$ we also have

$$
\mathcal{G}\left(x, \Gamma_{D}\right) \leq R^{*}+r^{*}
$$

and therefore (5.7) implies

$$
v(x) \leq a^{\prime} \leq a e^{\frac{1}{R^{*}+r^{*}} \ln \left(\frac{a^{\prime}}{a}\right) \mathcal{G}\left(x, \Gamma_{D}\right)}<a e^{-k \mathcal{G}\left(x, \Gamma_{D}\right)} .
$$

Next assume that $x \in \bar{\Omega}_{N} \backslash \bar{\omega}$ and let $x_{0}, \rho_{0}, \rho$ be as in $(\mathbf{H 3})$. Choose $y \in \partial \mathcal{B}_{\rho}\left(x_{0}\right)$ such that

$$
\mathcal{G}\left(y, \Omega_{D}\right)=\min _{z \in \partial \mathcal{B}_{\rho}\left(x_{0}\right):\left|z-x_{0}\right|=\rho} \mathcal{G}\left(z, \Omega_{D}\right) .
$$

Now note that the second condition of (H3) implies that the ray joining $x_{0}$ to $y$ is contained in $\overline{\mathcal{B}}_{\rho}\left(x_{0}\right)$. So, we have

$$
\mathcal{G}\left(x, \Omega_{D}\right) \leq \mathcal{G}\left(y, \Omega_{D}\right)+\rho_{0}+\rho .
$$

Therefore, from Lemma 2.2 using (5.12) and (5.7), we deduce that

$$
\begin{aligned}
& v(x) \leq \frac{\tilde{\varphi}\left(\rho_{0}\right)}{\tilde{\varphi}(\rho)} a e^{-k \mathcal{G}\left(y, \Omega_{D}\right)}=a e^{-k \mathcal{G}\left(y, \Omega_{D}\right)+\ln \left(\frac{\tilde{\varphi}\left(\rho_{0}\right)}{\tilde{\varphi}(\rho)}\right)} \\
& \quad \leq a e^{-k \mathcal{G}\left(y, \Omega_{D}\right)} e^{\ln \left(\frac{\tilde{\varphi}\left(\rho_{0}\right)}{\tilde{\varphi}(\rho)}\right)+k\left(\rho_{0}+\rho\right)}<a e^{-k \mathcal{G}\left(x, \Omega_{D}\right)} .
\end{aligned}
$$

So, from (5.10) and (5.13) $v$ is a free minimizer of the action functional. The exponential estimate $(5.5)$ follows from (5.10), (5.13) and

$$
\mathcal{G}\left(x, \Gamma_{D}\right) \leq \mathcal{G}\left(x, \Omega_{D}\right)+2 R^{*} .
$$

In Figure 2 we give an example of $\Omega, \Gamma_{D}, \Gamma_{N}$ that satisfies the assumptions in Theorem 5.2

\section{REFERENCES}

[1] F. Alessio, A. Calamai and P. Montecchiari. Saddle-type solutions for a class of semilinear elliptic equations. Adv. Differential Equations, 12:361-380, 2007. MR2305872 (2007m:35051)

[2] N. D. Alikakos and G. Fusco. Entire solutions for equivariant elliptic systems with variational structure. arXiv:0811.0106.

[3] N. D. Alikakos and G. Fusco. On an elliptic system with symmetric potential possessing two global minima. arXiv:0810.5009.

[4] N. D. Alikakos and G. Fusco. Entire solutions to nonconvex variational elliptic systems in the presence of a finite symmetry group. In Singularities in Nonlinear Evolution Phenomena and Applications-Proceedings, CRM Series, 2009, pp. 1-26. MR2528696

[5] H. Berestycki, L. A. Caffarelli and L. Nirenberg. Monotonicity for elliptic equations in unbounded Lipschitz domains. Comm. Pure Appl. Math. 50:1089-1111, 1997. MR1470317 (98k:35064)

[6] X. Cabré and J. Terra. Saddle-shaped solutions of bistable diffusion equations in all of $\mathbb{R}^{2 m}$. J. Eur. Math. Soc. 11:819-843, 2009. MR2538506 (2010j:35175)

[7] H. Dang, P. C. Fife and L. A. Peletier. Saddle solution of the bistable diffusion equation. $Z$. Angew. Math. Phys. 43:984-998, 1992. MR.1198672 (94b:35041) 
[8] D. Gilbarg and N. S. Trudinger. Elliptic Partial Differential Equations of Second Order. Springer-Verlag, 1983. MR737190 (86c:35035)

[9] P. Hess. On multiple positive solutions of nonlinear elliptic eigenvalue problems. Comm. Partial Differential Equations, 6:951-961, 1981. MR619753 (82j:35062)

[10] P. L. Lions. On the existence of positive solutions of semilinear elliptic equations. SIAM Review, 24:441-467, 1982. MR678562 (84a:35093)

[11] P. Omari and F. Zanolin. Infinitely many solutions of a quasilinear elliptic problem with an oscillatory potential. Comm. Partial Differential Equations, 21:721-733, 1996. MR.1391521 (97c:35068)

[12] G. Savaré. Regularity and perturbation results for mixed second order elliptic problems. Comm. Partial Differential Equations, 22:869-899, 1997. MR.1452171 (98d:35042)

[13] G. Stampacchia. Problemi al contorno misti per equazioni del calcolo delle variazioni. Ann. Matematica Pura Appl. 40:193-209, 1955. MR0089992(19:750c)

[14] J. Saint Raimond. On the multiplicity of the solutions of the equation $-\Delta u=\lambda f(u) . J$. Differential Equations, 180:65-88, 2002. MR1890598 (2003a:35141)

[15] J. Shi. Solution Set of Semilinear Elliptic Equations: Global Bifurcation and Exact Multyplicity. World Scientific Publ., 2008.

[16] J. Shi. Saddle solutions of the balanced bistable diffusion equation. Comm. Pure Appl. Math. 7:815-830, 2002. MR1894156 (2003b:35068)

[17] J. Smoller and A. Wasserman. Existence of positive solutions for semilinear elliptic equations in general domains. Arch. Ration. Mech. Anal. 98:229-249, 1987. MR867725 (88a:35101)

Dipartimento di Matematica Pura e Applicata, Università degli Studi di L'Aquila, Via Vetoio, Loc. Coppito, 67010 L'Aquila Italy

Dipartimento di Matematica Pura e Applicata, Università degli Studi di L'Aquila, Via Vetolo, Loc. Coppito, 67010 L'Aquila Italy

Dipartimento di Matematica Pura e Applicata, Università degli Studi di L'Aquila, Via Vetoio, Loc. Coppito, 67010 L'Aquila Italy 